\title{
Iron therapy in pregnant women with iron deficiency anemia: a meta-analysis
}

\author{
Anusha Natarajan*, Priyadarsini Baskaran
}

Department of Pharmacology, Jawaharlal Institute of Postgraduate Medical Education and Research, Puducherry, India

Received: 01 December 2020

Revised: 18 January 2021

Accepted: 20 January 2021

\section{*Correspondence:}

Dr. Anusha Natarajan,

Email: anushanatarajan29@gmail.com

Copyright: (c) the author(s), publisher and licensee Medip Academy. This is an open-access article distributed under the terms of the Creative Commons Attribution Non-Commercial License, which permits unrestricted non-commercial use, distribution, and reproduction in any medium, provided the original work is properly cited.

\begin{abstract}
Background: Pregnancy significantly increases the need for iron. The prevalence of anemia in pregnant women is high, affecting $41.8 \%$ of all pregnant women worldwide. In patients with low tolerance to oral iron, it is recommended to start them on parenteral iron therapy but with variable degree of efficacy. Hence this meta-analysis was done with the following aim. This study aimed to assess the efficacy of various iron preparations in pregnant women with iron deficiency anemia (IDA).

Methods: Randomised controlled trials (RCTs) (available as full free text) which included iron therapy in pregnant women with iron deficiency anemia were retrieved from electronic databases viz. PubMed, Google scholar and IndMed, with specific search terms. Qualities of RCTs were assessed using JADAD score and four RCTs with high score were included for analysis using RevMan 5.3 software. Outcome measures were change in hemoglobin levels and serum ferritin concentration after one month of therapy.

Results: In the four RCTs included, a total of 267 patients were treated with oral iron and 267 patients were treated with parenteral iron therapy. Change in the hemoglobin levels between the 2 groups had a standard mean difference of $0.73,95 \%$ CI (-0.05-1.52), with the p-value of 0.07 . To assess the change in the serum ferritin concentration a total of 188 patients in oral iron and 197 patients in parenteral iron therapy were included. There was a standard mean difference of $0.88,95 \%$ CI $(0.60-1.66)$, with a $p$ value of $<0.00001$.

Conclusions: In the present meta-analysis we found that oral and parenteral iron therapy showed similar efficacy in improving the hemoglobin level in pregnant women.
\end{abstract}

Keywords: Iron therapy, Pregnancy, Meta-analysis

\section{INTRODUCTION}

Pregnancy is a state of increased iron demand. Iron deficiency, which depends on the nutritional state of the patient, is the principal cause. ${ }^{1}$ This increased demand for iron subsequently places the mother and infant at risk of developing iron deficiency anemia (IDA), which can lead to gestational complications, as well as increased maternal and infant morbidity and mortality. ${ }^{2}$ Prevalence of iron deficiency anemia (IDA) in pregnant women worldwide is $41.8 \%$. $^{3}$ Oral iron therapy is routinely used to treat IDA in pregnant women. However, this can have gastrointestinal adverse effects which eventually lead to loss of adherence and decreased efficacy of iron therapy. ${ }^{4,5}$ In patients with low tolerance to oral iron, parenteral iron therapy is recommended. IV preparations can deliver a larger iron supply more rapidly than oral iron and, because of the route of administration, bypass the risk of gastrointestinal side effects. ${ }^{6}$

However, the efficacy of the different available parenteral iron compositions is variable. Hence this meta-analysis was done with the following objective. 


\section{Objectives}

The objectives were to compare the efficacy between oral and parenteral iron therapy in pregnant women with iron deficiency anemia (IDA).

\section{METHODS}

\section{Search strategy and study selection}

We conducted electronic searches in PubMed, Google scholar and IndMed to identify relevant articles. RCTs (available as full free text) with no restriction regarding to language, publication period, patient age was included in this study. The search term that was used was

\section{Search term}

1) Oral iron AND Iron deficiency anemia in pregnant females 2) parenteral iron AND iron deficiency anemia in pregnant females. Two independent reviewers (AN and PB) performed initial scrutiny of primary titles and abstracts (when available) to select potential full text articles for further scrutiny. When the title and abstract cannot be rejected by any reviewer, the full text of the article was obtained. Inclusion or exclusion of each study was determined by discussion and consensus between the two reviewers.

\section{Inclusion criteria}

Inclusion criteria were 1) types of participants. This review considers studies that include patients with iron deficiency anemia 2) types of intervention. This review considers studies that evaluate oral iron preparations and parenteral iron preparations in patients with iron deficiency anemia 3) types of outcomes. This review considers studies that included increase in hemoglobin levels after the use of either oral or parenteral iron preparations 4) types of studies. This review considers only randomized controlled trials for inclusion

\section{Data collection}

Quality of RCT and risk of bias was assessed using RevMan. Quality of the included RCTs were analyzed using JADAD score. Quantitative data was extracted from papers included in the review using the standardized data extraction tool from RevMan. ${ }^{7}$ The data extracted includes specific details about the interventions, populations, study methods and outcomes of significance to the review question and specific objectives.

\section{Statistical analysis}

Quantitative data were pooled in statistical meta-analysis using review manager 5.3. Effect sizes were expressed as weighted standard mean differences for continuous data and their $95 \%$ confidence intervals were calculated. Heterogeneity was assessed statistically using the Chisquare test. $\mathrm{p}<0.05$ was considered statistically significant.

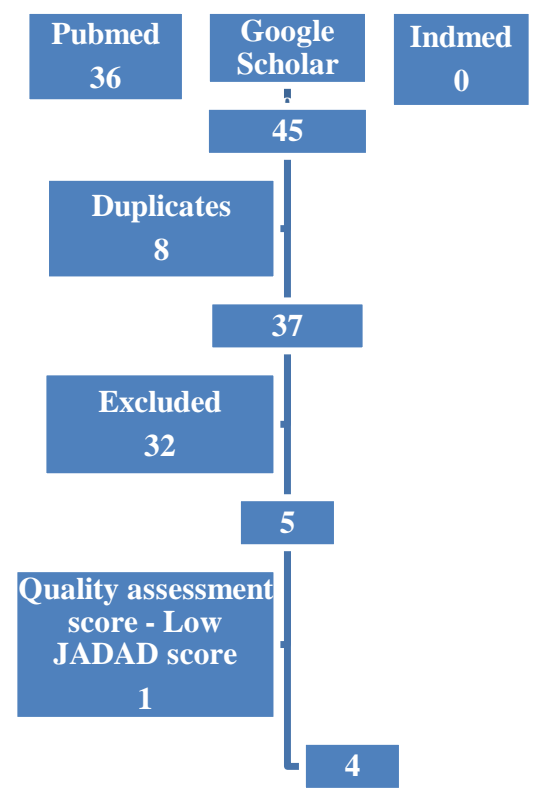

Figure 1: Method of article selection.

\section{RESULTS}

Here, $\mathrm{P}=0.07$, Figure 2 shows the change in the hemoglobin levels with oral iron therapy and parenteral iron therapy in pregnant women. This forest plot shows that there was no significant improvement in the hemoglobin levels between the two formulations.

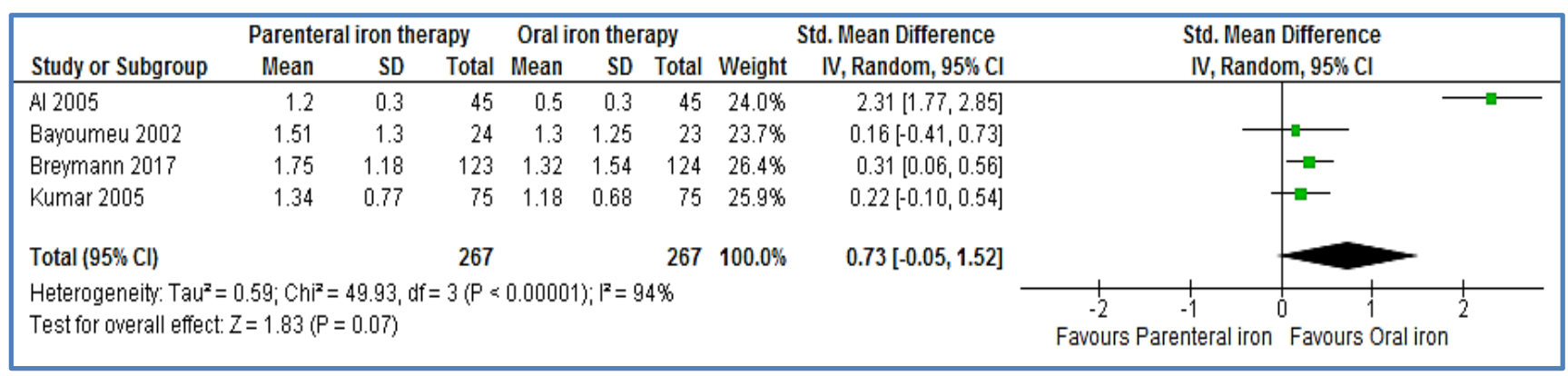

Figure 2: Change in hemoglobin levels with oral versus parenteral iron therapy in pregnant women with iron deficiency anemia. 


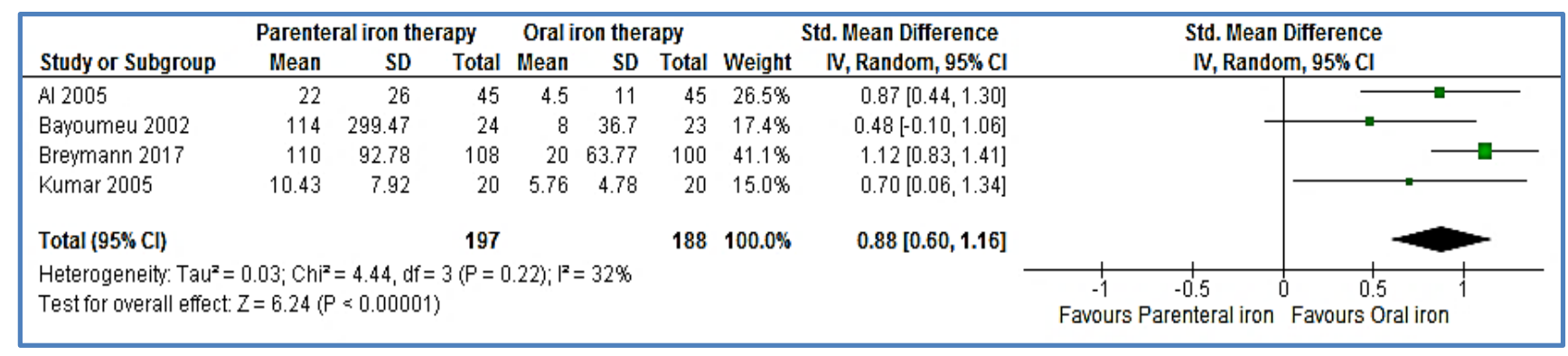

Figure 3: Change in serum ferritin levels with oral versus parenteral iron therapy in pregnant women with iron deficiency anemia.

$\mathrm{P}<0.00001$, Figure 3 shows the change in the serum ferritin levels with oral iron therapy and parenteral iron therapy in pregnant women. This shows that there was a statistically significant improvement in the serum ferritin levels in the oral iron therapy than parenteral iron therapy.

\section{DISCUSSION}

Iron deficiency anemia during pregnancy is common and deserves special attention because of its potential consequences. 8 In preparation for childbirth, Hb levels are known to rise as pregnancy progresses and peak before delivery. $^{2}$

The elevation in the hemoglobin levels were comparable in both the groups. Both parenteral and oral iron therapy was comparable to each other in improving the hemoglobin levels in pregnant women with iron deficiency anemia. Oral iron therapy had statistically significantly better efficacy in improving serum ferritin levels. In the study done by Breymann et al he showed that intravenous iron therapy was more appropriate option than first-line oral iron for rapid and effective anemia correction. In the study conducted by Bayoumeu et al demonstrated that parenteral iron therapy was efficacious with no serious adverse effects and also minimized the need for blood transfusion in the postpartum period. ${ }^{2,8}$

\section{Limitations}

We did not include the adverse effects of the two formulations for analysis. Studies documenting adverse effects of oral and parenteral iron therapy will provide added evidence to the present findings.

\section{CONCLUSION}

Oral and parenteral iron therapy showed similar efficacy. This suggests that wherever possible oral iron therapy is preferable owing to the lower cost and ease of administration and also being non-invasive administration, it will be preferred over the parenteral iron therapy.
Funding: No funding sources

Conflict of interest: None declared

Ethical approval: Not required

\section{REFERENCES}

1. Hercberg S, Galan P, Prual A, Preziosi P. Epidemiology of iron deficiency and iron deficiency anemia in the French population. Ann Biol Clin 1998;56:49-52.

2. Breymann C, Milman N, Mezzacasa A, Bernard R, Dudenhausen J. Ferric carboxymaltose vs. oral iron in the treatment of pregnant women with iron deficiency anemia: an international, open-label, randomized controlled trial (FER-ASAP). Journal of Perinatal Medicine. 2017;45(4).

3. de Benoist B, McLean E, Egli I, Cogswell M, (eds). Worldwide prevalence of anaemia 1993-2005: WHO global database on anaemia. 2008. Available at: http://whqlibdoc.who.int/publications/2008/9789241 596657_eng.pdf. Accessed on 20 June 2020.

4. Breymann C, Honegger C, Holzgreve W, Surbek D. Diagnosis and treatment of iron-deficiency anaemia during pregnancy and postpartum. Arch Gynecol Obstet. 2010;282:577-80.

5. Khalafallah AA, Dennis AE. Iron deficiency anaemia in pregnancy and postpartum: pathophysiology and effect of oral versus intravenous iron therapy. J Pregnancy. 2012;2012:630519.

6. Milman N. Prepartum anaemia: prevention and treatment. Ann Hematol. 2008;87:949-59.

7. Jadad Scale $>$ Score Calculator. Tools.farmacologiaclinica.info. Available from: http://tools.farmacologiaclinica.info/index.php?sid=3 6287. Accessed on 20 May 2020.

8. Bayoumeu F, Subiran-Buisset C, Baka N, Legagneur $\mathrm{H}$, Monnier-Barbarino P, Laxenaire M. Iron therapy in iron deficiency anemia in pregnancy: Intravenous route versus oral route. American Journal of Obstetrics and Gynecology. 2002;186(3):518-22.

Cite this article as: Natarajan A, Baskaran P. Iron therapy in pregnant women with iron deficiency anemia: a meta-analysis. Int J Basic Clin Pharmacol 2021;10:193-5. 